\title{
SOX12 contributes to the activation of the JAK2/STAT3 pathway and malignant transformation of esophageal squamous cell carcinoma
}

\author{
CHUNGUANG LI ${ }^{1 *}$, MAOLING ZHU ${ }^{2 *}$, JI ZHU ${ }^{1}$, QIJUE LU ${ }^{1}$, BOWEN SHI ${ }^{1}$, BIN SUN $^{3}$ and HEZHONG CHEN ${ }^{1}$ \\ ${ }^{1}$ Department of Thoracic Surgery, Changhai Hospital, Second Military Medical University, Shanghai 200438; \\ ${ }^{2}$ Department of Gastroenterology, Yangpu Hospital, Tongji University School of Medicine, \\ Shanghai 200090; ${ }^{3}$ Molecular Oncology Laboratory, Eastern Hepatobiliary Surgery Hospital, \\ Second Military Medical University, Shanghai 200438, P.R. China
}

Received May 15, 2020; Accepted October 6, 2020

DOI: $10.3892 /$ or.2020.7863

\begin{abstract}
As a crucial transcription factor, sex-determining region Y box 12 (SOX12) is closely related with tumorigenesis and malignant transformation in various malignant tumor types. To date, the specific function of SOX12 in esophageal squamous cell carcinoma (ESCC) has remained largely elusive and requires further investigation. The present study aimed to determine whether aberrant expression of SOX12 is associated with malignant development of ESCC. The expression level of SOX12 in ESCC cells and tissues was analyzed by RT-qPCR and western blotting. Short hairpin RNA (shRNA) targeting SOX12 was transfected into ESCC cells to knock down the expression of SOX12. Colony formation and Transwell assays were used to detect viability and mobility of ESCC cells. Signaling pathway-related proteins were assessed using western blot analysis and cellular immunofluorescence. Clinical prognosis data was analyzed by Kaplan-Meier and Cox logistic regression. The present results revealed that SOX12 was overexpressed in ESCC cells and tissues. Knockdown of the expression of SOX12 by shRNA inhibited the colony forming efficiency, migration and invasion capacities of ESCC
\end{abstract}

Correspondence to: Dr Bin Sun, Molecular Oncology Laboratory, Eastern Hepatobiliary Surgery Hospital, Second Military Medical University, 225 Changhai Road, Shanghai 200438, P.R. China

E-mail: sunbin05301984@aliyun.com

Dr Hezhong Chen, Department of Thoracic Surgery, Changhai Hospital, Second Military Medical University, 225 Changhai Road, Shanghai 200438, P.R. China

E-mail: drchenhz@163.com

${ }^{*}$ Contributed equally

Key words: sex-determining region $\mathrm{Y}$ box 12, transcription factor, esophageal squamous cell carcinoma, short hairpin RNA, Janus kinase 2/signal transducer and activator of transcription 3 signaling pathway cells in vitro. Recombinant protein of SOX12 could restore the aggressive phenotype of ESCC cells. Furthermore, knockdown of the expression of SOX12 inhibited the activation of the Janus kinase 2 (JAK2)/signal transducer and activator of transcription 3 (STAT3) signaling pathway by decreasing the expression of the JAK2/STAT3 signaling pathway. Recombinant protein of SOX12 could recover the activation of the JAK2/STAT3 signaling pathway. Analysis of the clinical data revealed that overexpression of SOX12 indicated shorter overall survival time (OS; $\mathrm{P}=0.0341$ ) and disease-free survival time (DFS; $\mathrm{P}=0.04$ ). Univariate and multivariate analysis revealed that overexpression of SOX12 was an independent factor for ESCC. In conclusion, SOX12 was revealed to serve a crucial function in sustaining the viability, as well as enhancing the motility of ESCC cells via activating the JAK2/STAT3 signaling pathway. Thus, SOX12 may potentially serve as a novel biomarker and candidate for the targeted treatment of ESCC.

\section{Introduction}

Esophageal cancer (EC) is a common human malignant tumor type with high incidence and mortality and the sixth leading cause of cancer-associated deaths worldwide (1-3). While esophageal adenocarcinoma (EAC) is the main histologic subtype in Western countries, esophageal squamous cell carcinoma (ESCC) remains the predominant form in Asian countries, accounting for more than $90 \%$ of EC (4). The development of early diagnostic and adjuvant treatment methods has provided a benefit for certain ESCC patients in recent decades. However, the poor prognosis of patients remains a major clinical problem (5). Recurrence and the ability to form metastasis are crucial characteristics of malignant compared with benign tumors, and are accountable for the mortality of a considerable proportion of affected patients. Tumor metastasis is multistep and complex biological process, including the spread of tumor cells from their primary location and disseminating through the bloodstream to eventually colonize in situ or in distant organs (6). Identification of effective regulators in every key regulatory step of malignant progression is anticipated to provide improvement for the diagnosis and treatment of ESCC. 
Sex-determining region Y-box 12 (SOX12) is well-known as a member of a transcription factor superfamily belonging to the high-mobility group, and has been revealed to perform key functions in embryonic development and the determination of cell fate $(7,8)$. Previous studies have also revealed that aberrant expression of SOX12 affects the biological behaviors of tumor cells and has a potential prognostic value in various type of tumors, such as liver (9), breast (10), and lung cancer (11), as well as renal carcinoma (12) and acute myeloid leukemia (13). However, the potential roles and specific regulatory mechanisms of SOX12 in ESCC remain unknown.

The purpose of this study was to investigate the role of SOX12 in the biological behavior of ESCC cells and its predictive value for clinical prognosis

\section{Materials and methods}

Patients and collection of tissue samples. Esophageal squamous cell carcinoma tissue and paired adjacent non-cancerous tissues $(n=56)$ were collected from patients who were diagnosed with primary ESCC and received radical esophageal surgery without preoperative radiotherapy, chemotherapy or biotherapy from August 2012 to September 2013 at Changhai hospital, Second Military Medical University (Shanghai, China). The median age of patients at the time of admission was 58 years, range 43-72 years. In total, 15 patients were women, and 41 were men. Total RNA from 56 pairs of tissue samples were extracted for RT-qPCR detection. Total protein from 6 pairs of tissue samples were extracted for western blot detection. All tissues (tumor tissue and paired para-cancerous tissue) were embedded in paraffin immediately after surgery. Each sample was confirmed by pathological diagnosis and was followed up until March 2018. The use of esophageal cancer tissues and clinical data was approved by the Biomedical Ethics Committee of the Second Military Medical University (Shanghai, China). Informed consent from each patient before surgery was obtained.

Immunohistochemistry (IHC). IHC was used to evaluate the expression level of SOX12 in paraffin-embedded tissues. All specimens had been fixed in 10\% buffered formalin and embedded in paraffin. The embedded tissues were cut into $4-\mu \mathrm{m}$-thick serial sections and stored at $4^{\circ} \mathrm{C}$. After deparaffinization, rehydration and antigen retrieval, each slide was blocked with $10 \%$ (w/v) normal goat serum (product no. SP KIT-B1; Fuzhou Maixin Biotech Co., Ltd.) at room temperature for $1 \mathrm{~h}$ and then incubated with anti-SOX12 primary antibody (dilution, 1:100; cat. no. SAB4502835; Sigma-Aldrich; Merck $\mathrm{KGaA}$ ) at $4^{\circ} \mathrm{C}$ overnight, followed by incubation with secondary antibodies (EliVision plus; product no. KIT-9901; Fuzhou Maixin Biotech Co., Ltd.) at room temperature for $1 \mathrm{~h}$ and diaminobenzidine dye (product no. DAB-0031 (20X); Fuzhou Maixin Biotech Co., Ltd.) at room temperature for 1-9 min. Counterstaining of the nucleus was performed with hematoxylin (product no. CTS-1099; Fuzhou Maixin Biotech Co., Ltd.) at room temperature for $10 \mathrm{~min}$. Negative controls were prepared by replacing the primary antibody with PBS. Images were obtained with light microscope at a magnification of x200. All tissues were reviewed by experienced pathologists. For each patient sample, the number and proportion of immunopositive cells was determined in 10 randomly selected high-power fields and immunoreactive scoring was performed. A score of $>4$ was considered as high expression.

Cell lines culture and vectors. The human ESCC cell lines Eca109, TE1 and the human normal esophageal epithelial cell line HEEC (used as control) were obtained from the Cell Bank of the Shanghai Institutes for Biological Sciences. The cells were maintained in DMEM medium supplemented with $10 \%$ FBS (HyClone; Cytiva) at $37^{\circ} \mathrm{C}$ in a $5 \% \mathrm{CO}_{2}$ incubator. The plasmids expressing short hairpin (sh)RNAs targeting SOX12 or scrambled shRNA (shRNA1: 5'-CATGGCGGA TTACCCGGACTA; shRNA2: 5'-TCCGCAGTCTTACGA GGAGTC; scrambled shRNA: 5'-GAGCTCTCTCTGCAC ATTCTT;) were purchased from Guangzhou RiboBio Co., Ltd. (product nos. stB0007967A-1-5, stB0007967B-1-5 and stB0007967C-1-5). Cell culture was performed in six well plates transfected with a total of $4 \mu \mathrm{g}$ shRNA using Lipofectamine ${ }^{\circledR} 2000$ transfection reagent (Invitrogen; Thermo Fisher Scientific, Inc.), used according to the manufacturer's protocol, that was added in each well when the cell density reached $50-60 \%$, and culture was continued at $37^{\circ} \mathrm{C}$ for 48-72 $\mathrm{h}$ according to the experimental requirements.

Recombinant human SOX12 protein (cat. no. H00006666-Q01; Abnova) and WP1066 (cat. no. HY-15312; MCE), a potent inhibitor of the JAK2/STAT3 signaling pathway were purchased and used according to the manufacturer's instructions. Recombinant human SOX12 protein $(10 \mu \mathrm{l})$ was co-cultured with shRNA1 in the colony formation, Transwell and cellular immunofluorescence assays. WP1066 (5 $\mu \mathrm{l})$, an inhibitor of the JAK2/STAT3 signaling pathway, was added to the cell culture medium in the colony formation, Transwell and western blotting assays. DMSO was used as a solvent control.

Colony formation assay. After transfection with the indicated plasmids, Eca109 and TE1 cells were collected and re-suspended in $1.5 \mathrm{ml}$ complete culture medium supplemented with $0.45 \%$ low-melting point agarose (Invitrogen; Thermo Fisher Scientific, Inc.). The cells $\left(1 \times 10^{3}\right)$ were inoculated in $35-\mathrm{mm}$ tissue culture dishes containing $1.5 \mathrm{ml}$ culture medium and agarose $(0.75 \%)$ on the bottom layer. The dishes were cultured for two weeks. Cell colonies were fixed with $4 \%$ formaldehyde solution for $15 \mathrm{~min}$ and stained with $0.005 \%$ crystal violet for 10-30 $\mathrm{min}$ at room temperature. The number of colonies with more than 10 cells were counted by light microscope at a magnification of x10. Experimental groups are representative of at least three independent experiments.

Migration and invasion assays. The migratory and invasive capacities of Eca109 and TE1 were assessed by using Transwell assays. A normal Transwell chamber was used for the migration assay, and a Transwell chamber whose membrane was pre-coated with Matrigel ${ }^{\circledR}$ (cat. no. 356231; Corning; BD Bisociences) was used for the invasion assay. For each group, $5 \times 10^{4}$ cells in $200 \mu 1$ serum-free medium were added to each upper chamber, while $500 \mu$ l DMEM containing $20 \%$ FBS was added to the lower chambers. After incubation for $48 \mathrm{~h}$, cells were fixed with $4 \%$ formaldehyde solution for $15 \mathrm{~min}$ and stained with $0.005 \%$ crystal violet for $10 \mathrm{~min}$ at room 
temperature and images were obtained by light microscope (magnification, x200). Experimental groups are representative of at least three independent experiments.

Reverse transcription-quantitative polymerase chain reaction $(R T-q P C R)$. Total RNA extraction from tissues and cell lines was performed with TRIzol ${ }^{\mathrm{TM}}$ reagent (cat. no. 15596026; Invitrogen; Thermo Fisher Scientific, Inc.) and then reverse-transcribed into complementary DNA immediately with the PrimeScript ${ }^{\mathrm{TM}}$ RT Reagent kit (cat. no. RR037B; Takara Bio, Inc.) according to the manufacturer's instructions. Fast SYBR ${ }^{\mathrm{TM}}$ Green Master Mix (cat. no. 4385612; Thermo Fisher Scientific;) was added to the PCR reaction mixture according to the manufacturers' protocols. The thermocycling conditions used for qPCR were as follows: $95^{\circ} \mathrm{C}$ for $2 \mathrm{~min} ; 45$ cycles at $95^{\circ} \mathrm{C}$ for $5 \mathrm{sec}, 55^{\circ} \mathrm{C}$ for $20 \mathrm{sec}$ and $72^{\circ} \mathrm{C}$, $30 \mathrm{sec}$. A melting curve was established under the following reaction conditions: $95^{\circ} \mathrm{C}$ for $15 \mathrm{sec}, 60^{\circ} \mathrm{C}$ for $1 \mathrm{~min}$ and $95^{\circ} \mathrm{C}$ for $15 \mathrm{sec}$. The experiment was performed in triplicate. GAPDH was used as internal control. The $2^{-\Delta \Delta \mathrm{Cq}}$ method (14) was conducted to calculate the relative expression of the genes. The primer sequences used were as follows: SOX12 (NM_006943.3) forward, 5'-AGCACCCGTGTGACTCTT TCC-3' and reverse, 5'-AGCAGAACCAAGCCCTGTCTC-3'; GAPDH (NM_001256799.1) forward, 5'-CACCCACTCCTC CACCTTTG-3' and reverse, 5'-CCACCACCCTGTTGCTGT AG-3'.

Western blot analysis. Total protein from tissues and cell lines was extracted by using radioimmunoprecipitation assay lysis buffer (product no. P0013B; Beyotime Institute of Biotechnology). The total protein concentration was determined using the BCA method. A total of $30 \mu \mathrm{g}$ of protein was separated by SDS-PAGE (10\%) and transferred to a PVDF membrane. Non-specific binding sites were blocked by incubating with TBST $(0.1 \%$ Tween 20$)$ containing $5 \%(\mathrm{w} / \mathrm{v})$ non-fat dried milk for $1 \mathrm{~h}$ at room temperature. The antibodies used were as follows: Anti-SOX12 primary antibody (product no. SAB1412152; dilution, 1:1,000; Sigma-Aldrich; Merck KGaA), anti-JAK primary antibody (product code ab108596; dilution, 1:5,000), anti-phosphorylated (p)-JAK at Tyr1007+1008 primary antibody (product code ab32101; dilution, 1:2,000), anti-STAT3 primary antibody (product code ab68153; dilution, 1:1,000), anti-p-STAT3 (phosphorylated at Tyr705) antibody (product code ab76315; dilution, 1:5,000), anti-GAPDH antibody (product code ab9482; dilution, 1:5,000; all from Abcam) and goat anti-rabbit immunoglobulin G H\&L (cat. no. ab205718; dilution, 1:5,000; Abcam). Signals were visualized by ECL chemiluminescence (cat. no. 34095, Thermo Fisher Scientific, Inc.). The results were evaluated with Quantity One software (v4.6.6; Bio-Rad Laboratories, Inc.).

Cellular immunofluorescence. ESCC cell lines Eca109 and TE1 were seeded onto coverslips in six-well cell culture plates at $2 \times 10^{4}$ cells/well with complete medium and transfected with different shRNAs as aforementioned. After culture for $48 \mathrm{~h}$, the cells were rinsed three times with PBS carefully and then fixed with $4 \%$ formaldehyde solution for $15 \mathrm{~min}$ at room temperature. Cells were then permeabilized with Triton X-100 solution $(1 \% \mathrm{v} / \mathrm{v})$ for $10 \mathrm{~min}$ and blocked with non-immune goat serum for $1 \mathrm{~h}$ at room temperature. Cells were incubated with various antibodies as follows: SOX12 (product no. HPA055052; 1:100 dilution; Sigma-Aldrich; Merck KGaA), and the aforementioned antibodies p-JAK (phosphorylated at Tyr1007+1008; 1:100 dilution) and p-STAT3 (phosphorylated at Tyr705; dilution, $1: 100$ ) at $4^{\circ} \mathrm{C}$ overnight. Cells were then rinsed with PBS and incubated with cyanine 3-labeled (product no. A0516, 1:500 dilution) and Alexa 488-labeled (product no. A0423; 1:500 dilution) secondary antibodies (Beyotime Beyotime Institute of Biotechnology) for $1 \mathrm{~h}$ at room temperature, and stained with DAPI $(1 \mu \mathrm{g} / \mathrm{ml}$; Sigma-Aldrich; Merck $\mathrm{KGaA}$ ) for $5 \mathrm{~min}$ at room temperature. Images were obtained using a fluorescence microscope (magnification, x200).

Statistical analysis. Data analyses were calculated by SPSS 18.0 statistical software (SPSS, Inc.) and values are expressed as the mean \pm standard deviation. ANOVA and Dunnett's test were used for comparing the mean of multiple experimental groups and that of the control group. The chi-square test and Spearman's rank correlation were used to analyze the correlation between gene expression and clinical pathological data. Survival analysis was performed using the Kaplan-Meier method and significant differences in overall survival (OS) and disease-free survival (DFS) was determined with the log-rank test. Univariate and multivariate analyses were performed using Cox's proportional hazard model. Differences between multiple groups were analyzed using one-way analysis of variance. $\mathrm{P}<0.05$ was considered to indicate a statistically significant difference. The experimental data were representative of three independent experiments.

\section{Results}

SOX12 is aberrantly upregulated in ESCC tissues and cell lines. In the present study, the protein level of SOX12 in 56 ESCC samples and adjacent non-cancerous tissues was examined by IHC. The results indicated that SOX12 was upregulated in ESCC tissues compared with adjacent non-cancerous tissues in 38 out of 56 cases (67.9\%; Fig. 1A and B). Then 6 pairs of fresh tissue samples were collected and total proteins were extracted. The protein level of SOX12 in ESCC tissues and adjacent non-cancerous tissues was detected by western blot analysis. The results revealed that the protein levels of SOX12 were markedly upregulated in ESCC tissue samples compared with those in the corresponding non-cancerous tissues (Fig. 1C). In addition, the protein and mRNA expression of SOX12 in ESCC cell lines was evaluated by western blot and RT-qPCR analysis, respectively. Human normal esophageal epithelial cell line (HEEC) was used as a control. As presented in Fig. 1D and E, compared with that in the HEEC human normal esophageal epithelial cell line, SOX12 was significantly overexpressed in both Eca109 and TE1. Collectively, these results indicated that SOX12 was aberrantly overexpressed in ESCC cell lines and tissue samples.

Knockdown of SOX12 inhibits the colony formation, migration and invasion of ESCC cell lines. To explore the function of SOX12 which regulates the biological characteristics of ESCC cells, three vectors carrying shRNA targeting SOX12, 
A

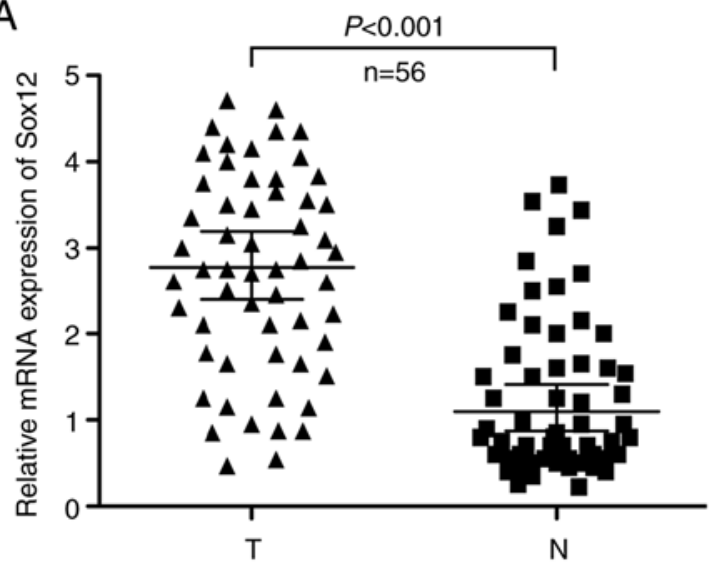

B

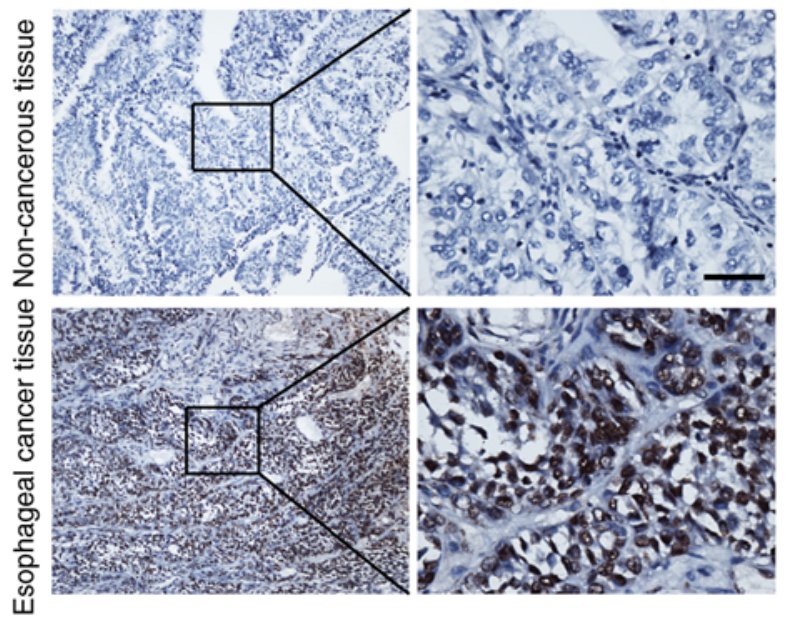

C
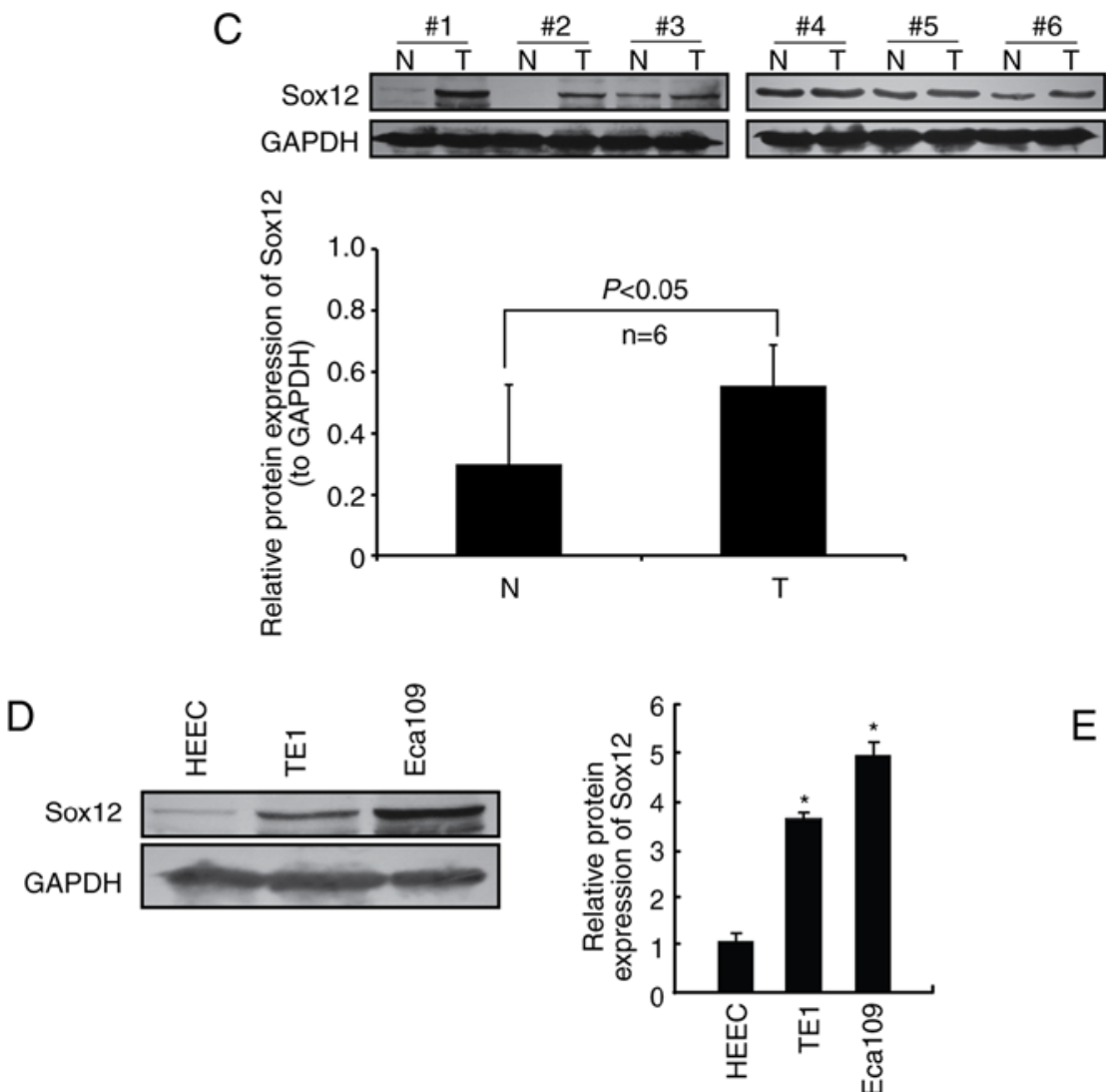

E

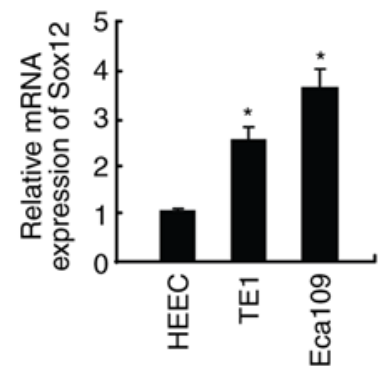

Figure 1. SOX12 is highly expressed in ESCC. (A) RT-qPCR was performed to evaluate the mRNA expression levels of SOX12 in 56 pairs of cancer and para-cancerous tissue. (B) Representative fields of SOX12 expression in ESCC samples (scale bar, $100 \mu \mathrm{m}$ ). (C) Protein expression levels of SOX12 in ESCC samples ( $\mathrm{n}=6$ ). (D) Protein expression levels of SOX12 in ESCC cell lines. (E) RT-qPCR assays were conducted to evaluated the mRNA level of SOX12. ${ }^{*} \mathrm{P}<0.05$ vs. HEEC. SOX12, sex-determining region Y box 12; ESCC, esophageal squamous cell carcinoma; RT-qPCR, reverse transcription-quantitative PCR; $\mathrm{N}$, non-tumor tissue; $\mathrm{T}$, tumor tissue.

shRNA1, shRNA2 and shRNA3, were constructed and respectively transfected into Eca109 and TE1 cells. Two of the vectors, shRNA1 and shRNA2, were more effective at knocking down the expression of SOX12 (Fig. 2), as determined by RT-qPCR and western blot analysis. Colony formation and Transwell assays were then performed to examine the neoplastic capacity and motility of SOX12-silenced Eca109 and TE1 cells. The results revealed that downregulation of SOX12 could inhibit the viability and motility capacities of ESCC cells in vitro (Fig. 3). When recombinant SOX12 $(10 \mu \mathrm{M})$ was co-cultured in transfected cells, the proliferation and motility of ESCC cells could be restored (Fig. S1A and B). It was therefore revealed that overexpression of SOX12 was closely related with the malignant biological behavior of ESCC cells.

Knockdown of SOX12 expression suppresses the JAK2/STAT3 signaling pathway in ESCC cells. Mutational activation of the JAK2/STAT3 signaling pathway is responsible for the malignant transformation and progression of various types of tumors (15). In this study, the protein expression levels of JAK2/STAT3 
A

Eca109

TE1
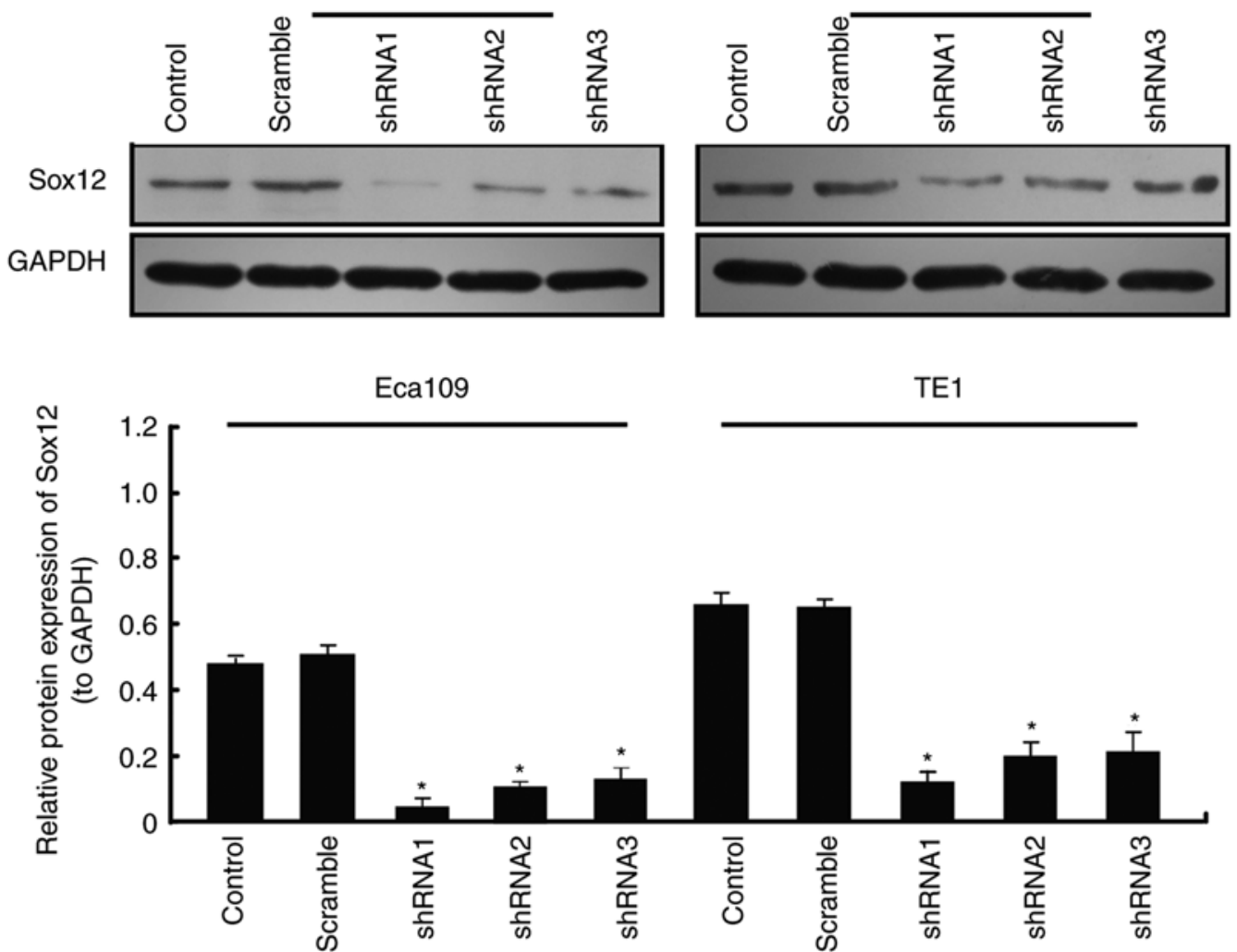

B
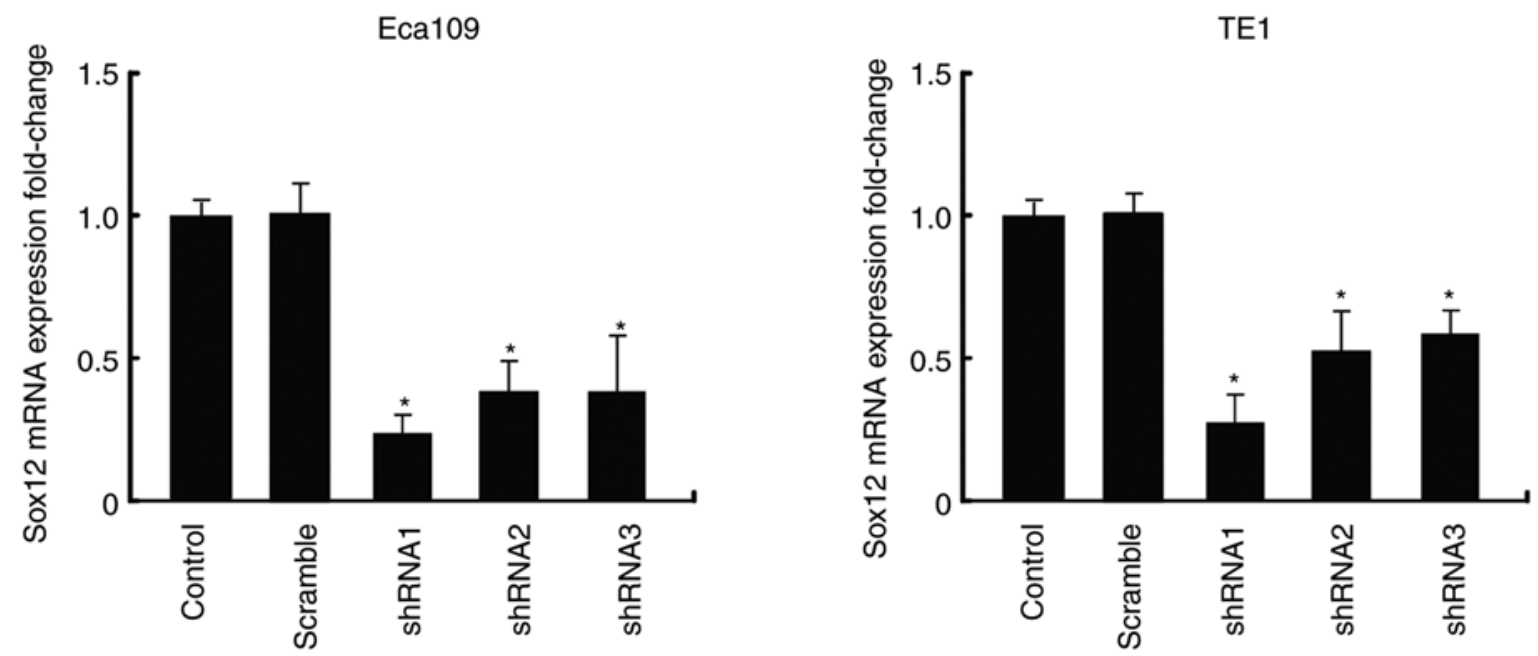

Figure 2. Detection of knockdown efficiency of shRNA plasmid. (A) Detection of SOX12 protein expression levels after transfection with different shRNA plasmids in ESCC cell lines. (B) RT-qPCR was carried out to detect the mRNA expression level of SOX12 after transfection with different shRNA plasmids in ESCC cell lines. " $\mathrm{P}<0.05$ vs. the control. shRNA, short hairpin RNA; SOX12, sex-determining region Y box 12; ESCC, esophageal squamous cell carcinoma; RT-qPCR, reverse transcription-quantitative PCR.

signaling proteins were measured by immunofluorescence and western blot assay. The results revealed that knockdown of SOX12 in ESCC cells decreased the levels of p-JAK2 $2^{\text {Tyr1007+1008 }}$ and p-STAT3 ${ }^{\text {Tyr705 }}$ (Fig. 4), which could be reversed by co-culture with recombinant SOX12 $(10 \mu \mathrm{M})$ (Fig. S1C). In addition, WP1066 $(5 \mu \mathrm{M})$ could inhibit colony formation and motility of ESCC cells in vitro (Fig. S2). Thus, it may be inferred that SOX12 promotes the malignant biological behavior of ESCC cells, including proliferation, invasion and migration, at least in part through activation of JAK2/STAT3 signaling pathway.
Increased expression of SOX12 predicts poor prognosis for ESCC patients. The correlation between SOX12 and clinicopathological factors of patients with ESCC was then evaluated. Statistical analysis indicated no significant difference in any of the clinicopathological characteristics of ESCC patients, including sex, age, degree of differentiation, tumor invasion and lymph node metastasis, between patients with high and low expression of SOX12 in their ESCC tissues (Table I). To explore the effect of SOX12 expression (high vs. low) on the clinical prognosis of ESCC patients, Kaplan-Meier survival 

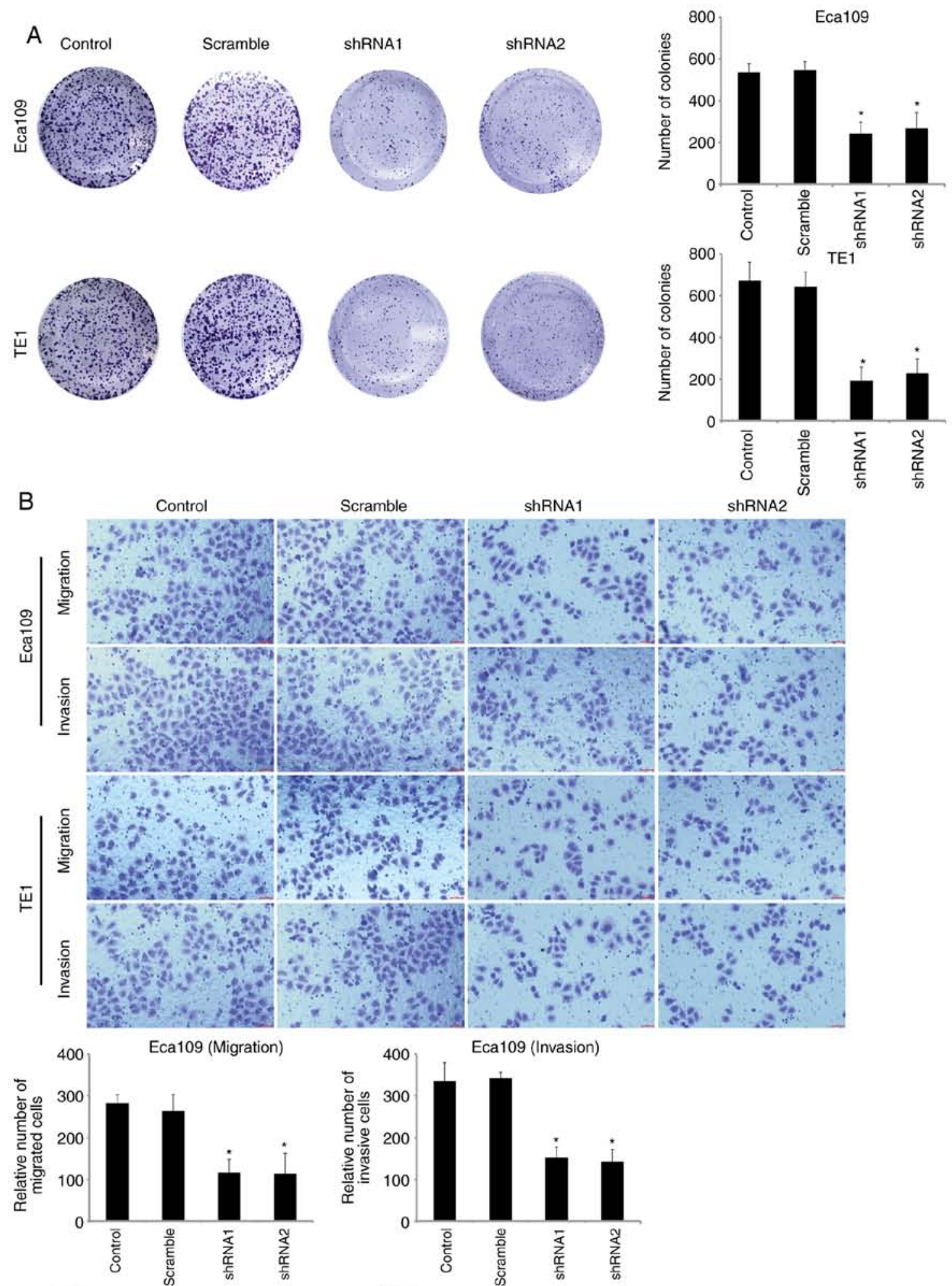

Scramble
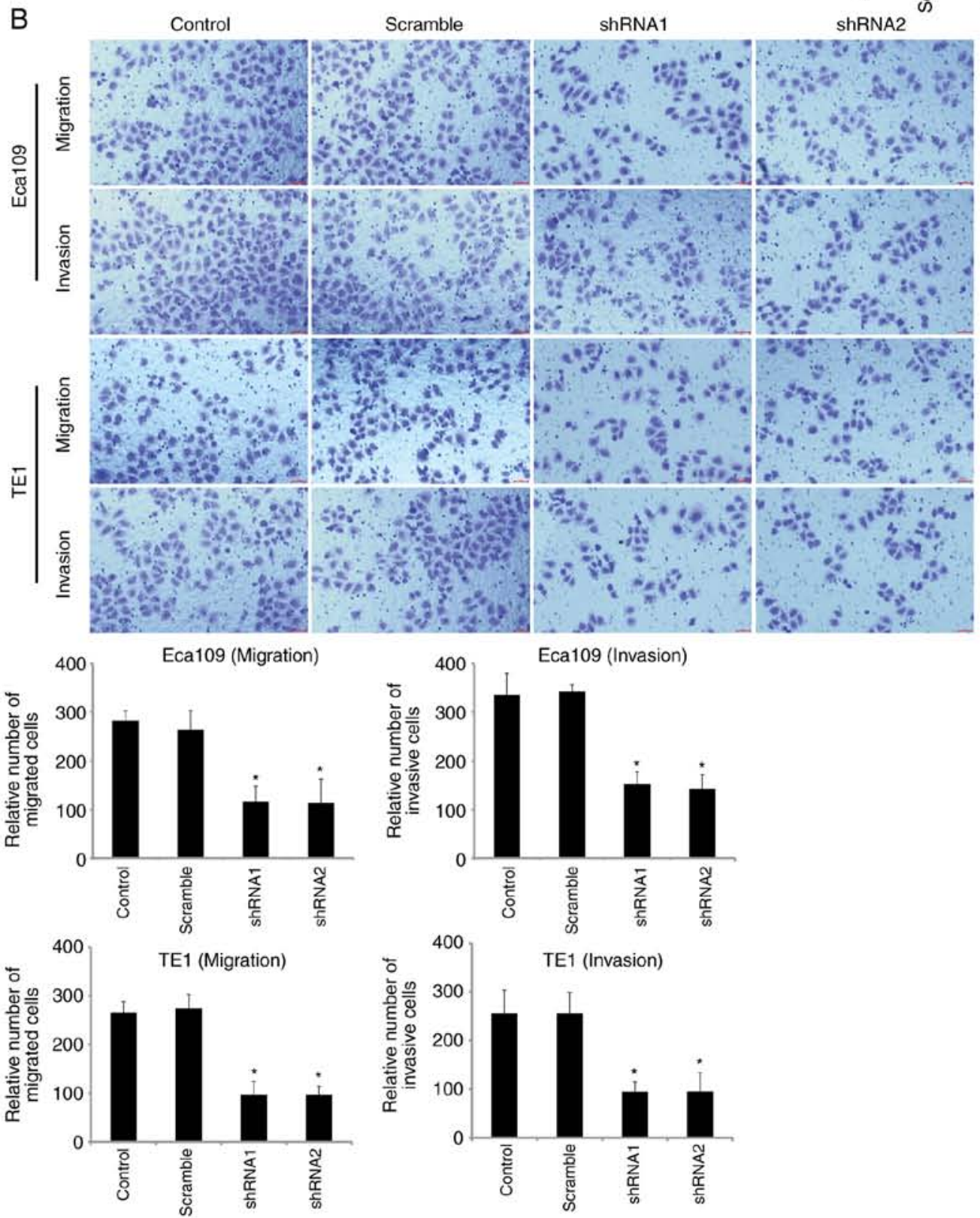

Figure 3. Downregulation of the expression of SOX12 inhibits the viability and motility of ESCC cells. (A) Downregulation of SOX12 decreased the colony formation rate of ESCC cells. (B) The effects of SOX12 knockdown on the migration and invasion abilities of ESCC cell lines were assessed by Transwell chamber assays. " $\mathrm{P}<0.05$ vs. control. SOX12, sex-determining region Y box 12; ESCC, esophageal squamous cell carcinoma; shRNA, short hairpin RNA. 

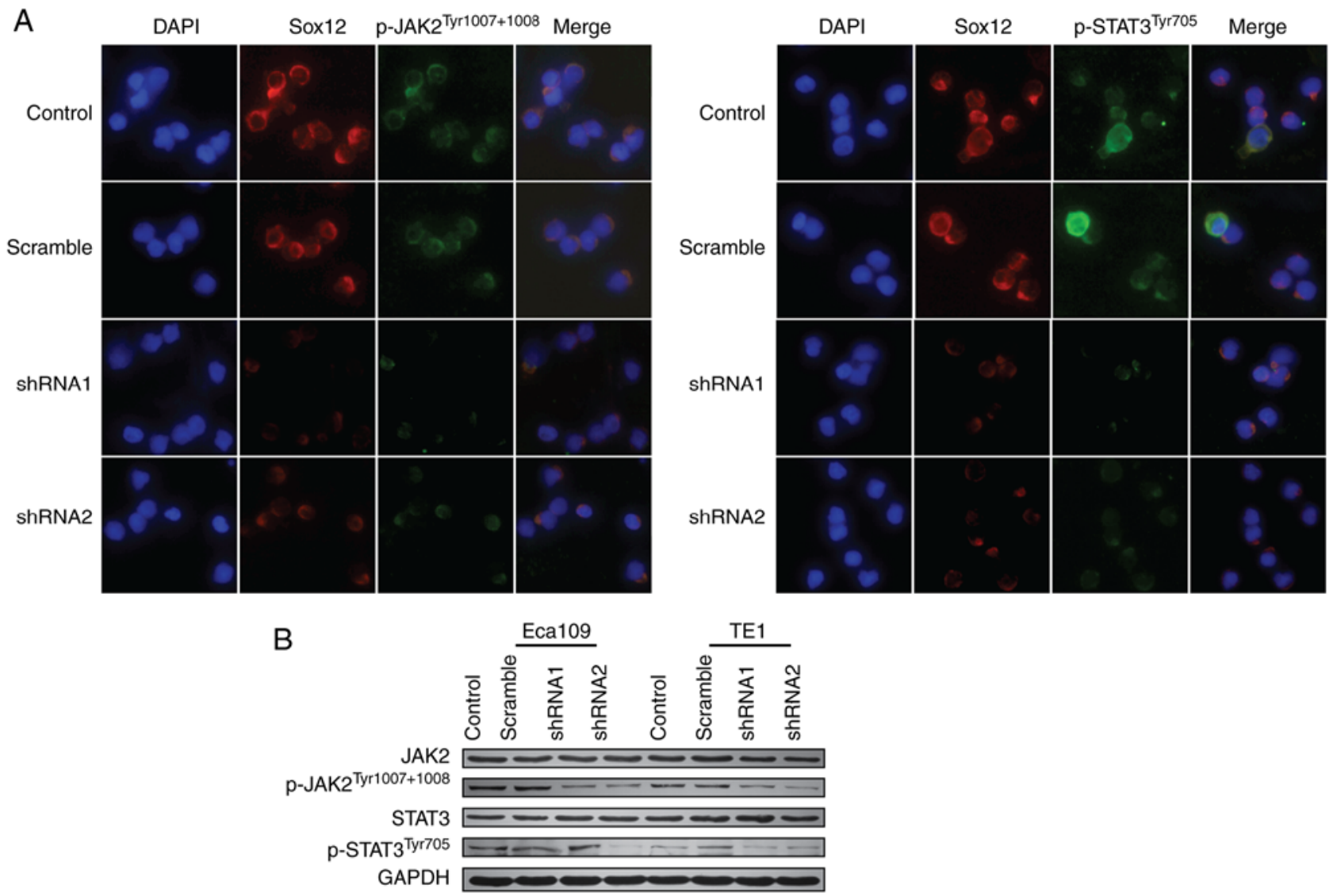

Figure 4. SOX12 promotes the proliferation, migration and invasion of ESCC cells by activating the JAK2/STAT3 signaling pathway. (A) SOX12,

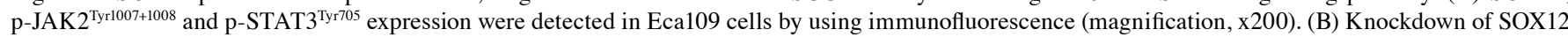
significantly downregulated the protein expression of p-JAK2 $2^{\text {Tyr1007+1008 }}$ and p-STAT3 ${ }^{\text {Tyr705 }}$ in ESCC cell lines. SOX12, sex-determining region Y box 12; ESCC, esophageal squamous cell carcinoma; JAK2, Janus kinase 2; STAT3, signal transducer and activator of transcription 3; p-, phosphorylated; shRNA, short hairpin RNA.
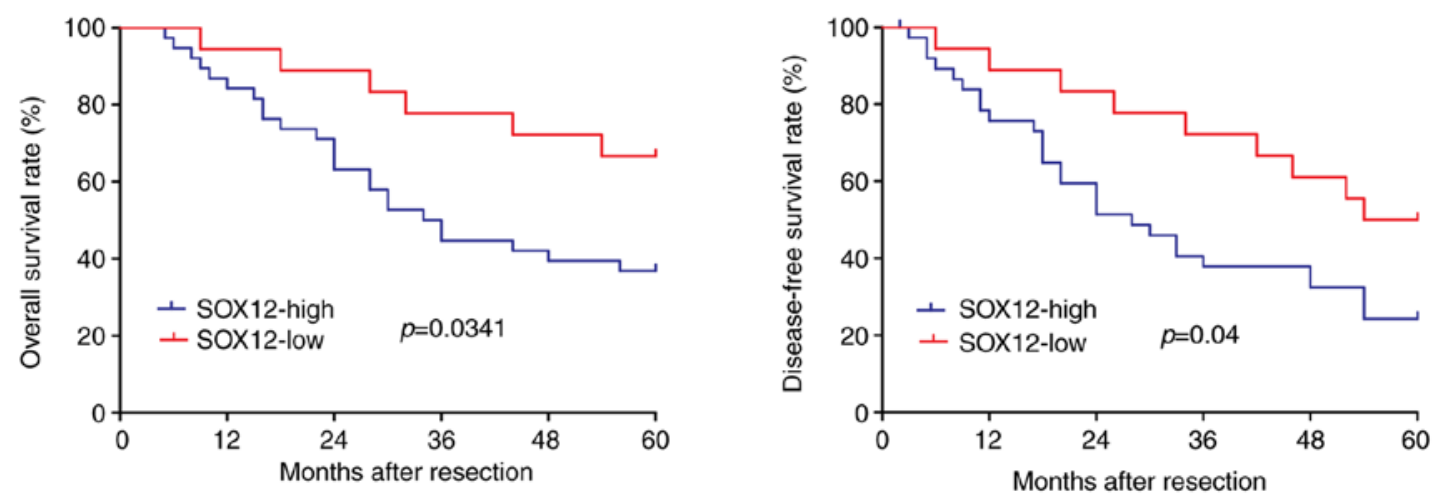

Figure 5. Overexpression of SOX12 is associated with the poor prognosis of ESCC patients. Overall survival rate and disease-free survival rate in 56 patients who underwent resection were stratified based on SOX12 expression levels (high or low). High expression of SOX12 was associated with a poorer overall $(\mathrm{P}=0.0341)$ and disease-free survival $(\mathrm{P}=0.04)$. SOX12, sex-determining region $\mathrm{Y}$ box 12 .

analysis was used. As presented in Fig. 5, high expression of SOX12 was associated with lower OS $(\mathrm{P}=0.0341)$ and DFS $(\mathrm{P}=0.04)$ compared with low expression of SOX12. Accordingly, overexpression of SOX12 was closely associated to the poor prognosis and survival of patients.

Analysis of the clinical data by univariate logistic regression using Cox's proportional hazards model indicated that SOX12 is a significant prognostic factor for ESCC patients $(\mathrm{P}=0.016$; Table II). Multivariate logistic regression analysis revealed that SOX12 is an independent prognostic factor for ESCC patients $(\mathrm{P}=0.044$; Table II). The results of clinical analysis revealed that overexpression of SOX12 may affect the malignant characteristics of ESCC and poor prognosis.

\section{Discussion}

ESCC is the main cause of cancer-related deaths in China (16). Radical resection remains the first choice of treatment for 
Table I. Association between SOX12 expression and ESCC clinicopathological features.

\begin{tabular}{lrrrrr}
\hline & \multicolumn{5}{c}{$\begin{array}{c}\text { SOX12 } \\
\text { expression }\end{array}$} \\
\cline { 3 - 4 } Variables & $\mathrm{n}$ & High & Low & $\chi^{2}$ & P-value \\
\hline Sex & & & & 0.282 & 0.596 \\
$\quad$ Male & 41 & 27 & 14 & & \\
Female & 15 & 11 & 4 & & \\
Age (years) & & & & 0.136 & 0.712 \\
$\leq 60$ & 26 & 17 & 9 & & \\
$>60$ & 30 & 21 & 9 & & \\
Differentiation & & & & 2.994 & 0.224 \\
Grade 1 & 17 & 9 & 8 & & \\
Grade 2 & 32 & 23 & 9 & & \\
Grade 3 & 7 & 6 & 1 & & \\
p-T & & & & 1.309 & 0.253 \\
T1/2 & 19 & 11 & 8 & & \\
T3 & 37 & 27 & 10 & & \\
p-N & & & & 0.083 & 0.773 \\
N0 & 17 & 12 & 5 & & \\
N1/2 & 39 & 26 & 13 & & \\
\hline
\end{tabular}

SOX12, sex-determining region $\mathrm{Y}$ box 12; ESCC, esophageal squamous cell carcinoma.

ESCC (17). Postoperative recurrence and metastasis are the key factors limiting the long-term prognosis of patients (18). Elucidation of the potential mechanisms of tumor progression may enhance the current understanding of ESCC and lead to the identification of valuable biomarkers for diagnosis and targets for treatment.

A previous study has indicated that SOX12, a multifunctional nuclear transcription factor, serves as a crucial role in embryonic development and cell-fate determination (8). A recent study indicated that SOX12 may functionally contribute to maintain stem-like characteristics of hepatocellular carcinoma (HCC) cells (19). Furthermore, SOX12 was reported to be responsible for metastasis via activation of the epithelial-mesenchymal transition process in HCC (20). However, at present, the understanding of the function of SOX12 in ESCC remains limited.

In the present study, SOX12 was revealed to be significantly upregulated in ESCC cell lines and tissues. These results revealed that SOX12 could acts as an oncogene and its upregulation may participate in the initial tumorigenesis as well as the malignant development of human cancer, including HCC (9), breast cancer (10), lung cancer (11), renal cancer (12) and acute myeloid leukemia (13).

The role of SOX12 in ESCC cells was then explored using knockdown experiments. The present findings indicated that downregulation of SOX12 significantly inhibited the proliferation and motility of ESCC cells in vitro. Co-culture of recombinant SOX12 in transfected cells could recover the proliferation and motility abilities of ESCC cells. These results
Table II. Univariate and Multivariate Cox's proportional hazard models $(n=56)$.

\begin{tabular}{lccc}
\hline Term & $\begin{array}{c}\text { Risk } \\
\text { ratio }\end{array}$ & $\begin{array}{c}95 \% \text { Confidence } \\
\text { interval }\end{array}$ & P-value \\
\hline $\begin{array}{l}\text { Univariate } \\
\text { Grade }\end{array}$ & 1.372 & $0.742-2.594$ & 0.229 \\
p-T & 1.775 & $0.825-3.767$ & 0.106 \\
P-N & 1.546 & $0.797-3.141$ & 0.157 \\
SOX12 & 2.242 & $1.161-4.673$ & 0.016 \\
Multivariate & & & \\
Grade & 1.423 & $0.765-2.903$ & 0.257 \\
p-T & 1.814 & $0.894-3.885$ & 0.142 \\
P-N & 1.606 & $0.807-3.302$ & 0.183 \\
SOX12 & 2.105 & $1.073-4.353$ & 0.044 \\
\hline
\end{tabular}

SOX12, sex-determining region Y box 12 .

indicated that SOX12 has a significant effect on the malignant biological behavior of ESCC cells.

Furthermore, clinical data revealed that high expression of SOX12 in ESCC tissues was closely associated to the poor prognosis of patients. Overexpression of SOX12 indicated shorter OS time ( $\mathrm{P}=0.0341)$ and $\mathrm{DFS}$ time $(\mathrm{P}=0.04)$. The expression of SOX12 in esophageal cancer vs. adjacent non-cancerous tissues was higher in $67.9 \%$ of cases. Univariate logistic regression analysis using Cox's proportional hazards model revealed that SOX12 could be a significant prognostic factor for ESCC patients $(\mathrm{P}=0.016)$. Furthermore, multivariate logistic regression analysis indicated that $\mathrm{SOX} 12$ was an independent prognostic factor in ESCC patients $(\mathrm{P}=0.044)$. However, there were no significant differences in the clinicopathological characteristics between patients with high and low expression of SOX12. This result may be due to two reasons. First, the small size of the cohort. Second, post-translational modification (such as methylation modification) may be involved and play a key role. In a subsequent study, the clinical sample size will be expanded, and further investigation will be performed to verify the results. In conclusion, these results indicated that SOX12 was associated with malignant transformation of ESCC and poor prognosis.

As a multifunctional nuclear transcription factor, SOX12 is able to participate in the regulation of multiple signaling pathways, including WNT/T-cell factor (21). In our pre-experiment (data not shown), several signaling pathways such as PI3K/AKT, MAPK/ERK, Wnt/ $\beta$-catenin and JAK2/STAT3 had been detected. Among all the signaling pathway proteins we detected, JAK2/STAT3 showed the most significant difference. This pathway can regulate several cellular behaviors by activating receptors or intracellular kinases to the nucleus to regulate gene transcription (22). In recent studies, the JAK2/STAT3 signaling pathway has been revealed to play a crucial role in proliferation, motility and stemness in cancer cells. USP9X positively regulated the JAK2/STAT3 pathway to promote the malignant progression of liver cancer cells (15). miR-375 inhibited the 
stemness of breast cancer cells by blocking the JAK2/STAT3 signaling (23). In the present study, knockdown of SOX12 in ESCC cells decreased the levels of p-JAK2 $2^{\text {Tyr1007+1008 }}$ and

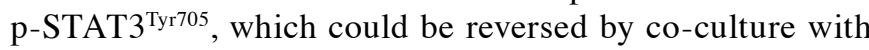
recombinant SOX12 $(10 \mu \mathrm{M})$. WP1066 $(5 \mu \mathrm{M})$, a novel potent inhibitor of the JAK2/STAT3 signaling pathway (24), could inhibit colony formation and motility of ESCC cells in vitro. The present results indicated that SOX12 could increase the colony formation rate, mobility of ESCC cells through activation of the JAK2/STAT3 signaling pathway which has been intensely investigated in various cancer types (25). Mutational activation of the JAK2/STAT3 is responsible for the malignant transformation and progression of several types of tumor (26-29). The present findings indicated that SOX12 could serve a crucial function in maintaining the malignant biological phenotype of ESCC cells via activating the JAK2/STAT3 signaling pathway. In a subsequent study, we will further explore the exact mechanism between SOX12 and ESCC, since more in vitro and in vivo experimental evidence is required to be investigated.

Collectively, the present findings revealed that SOX12 could serve as an oncogenic factor in ESCC. Aberrantly high levels of SOX12 were indicated to be associated with malignant biological behavior exhibited in ESCC cells and were revealed to be an independent unfavorable prognostic factor for patients with ESCC. SOX12 may become a novel prognostic biomarker and candidate for the targeted therapy of ESCC.

\section{Acknowledgements}

Not applicable.

\section{Funding}

Not applicable.

\section{Availability of data and materials}

The datasets used and/or analyzed during the current study are available from the corresponding author on reasonable request.

\section{Authors' contributions}

CL and MZ collected, analyzed and interpreted the data as well as were major contributors in writing the manuscript. JZ provided the majority of statistical analysis as well as provided the figures and tables for the manuscript. QL and BoS collected a large amount of data for the dataset. $\mathrm{HC}$ and BiS oversaw the analysis of the dataset, provided guidance in creating the dataset and manuscript, and were major contributors in writing the manuscript. All authors have read and approved the manuscript in its current state.

\section{Ethics approval and consent to participate}

The present study was approved by the Biomedical Ethics Committee of the Second Military Medical University (Shanghai, China). Written informed consent was obtained from all patients.

\section{Patient consent for publication}

Not applicable.

\section{Competing interests}

The authors declare that they have no competing interests.

\section{References}

1. Kamangar F, Dores GM and Anderson WF: Patterns of cancer incidence, mortality, and prevalence across five continents: Defining priorities to reduce cancer disparities in different geographic regions of the world. J Clin Oncol 24: 2137-2150, 2006.

2. Wen SW, Zhang YF, Li Y, Liu ZX, Lv HL, Li ZH, Xu YZ, Zhu YG and Tian ZQ: Association of miR-21 with esophageal cancer prognosis: A meta-analysis. Genet Mol Res 14: 6578-6582, 2015.

3. Zhao K, Chen BJ, Chen ZG, Zhang YJ, Xu D and Liu Q: Effect of miR-503 down-regulation on growth and invasion of esophagus carcinoma and related immune function. Med Sci Monit 21: 3564-3569, 2015

4. Domper Arnal MJ, Ferrández Arenas Á and Lanas Arbeloa Á: Esophageal cancer: Risk factors, screening and endoscopic treatment in Western and Eastern countries. World J Gastroenterol 21: 7933-7943, 2015.

5. Xu Y, Yu X, Chen Q and Mao W: Neoadjuvant versus adjuvant treatment: Which one is better for resectable esophageal squamous cell carcinoma? World J Surg Oncol 10: 173, 2012.

6. Han TS, Hur K, Xu G, Choi B, Okugawa Y, Toiyama Y, Oshima H, Oshima M, Lee HJ, Kim VN, et al: MicroRNA-29c mediates initiation of gastric carcinogenesis by directly targeting ITGB1. Gut 64: 203-214, 2015.

7. Dy P, Penzo-Mendez A, Wang H, Pedraza CE, Macklin WB, and Lefebvre V: The three SoxC proteins-Sox4, Sox 11 and Sox12-exhibit overlapping expression patterns and molecular properties. Nucleic Acids Res 36: 3101-3117, 2008.

8. Hoser M., Potzner MR, Koch JM, Bosl MR, Wegner M and Sock E: Sox 12 deletion in the mouse reveals nonreciprocal redundancy with the related Sox4 and Sox11 transcription factors. Mol Cell Biol 28: 4675-4687, 2008.

9. Huang W, Chen Z, Shang X, Tian D, Wang D, Wu K, Fan D and Xia L: Sox12, a direct target of FoxQ1, promotes hepatocellular carcinoma metastasis through up-regulating Twist1 and FGFBP1. Hepatology 61: 1920-1933, 2015.

10. Ding H, Quan H, Yan W and Han J: Silencing of SOX12 by shRNA suppresses migration, invasion and proliferation of breast cancer cells. Biosci Rep 36: e00389, 2016.

11. Wang L, Hu F, Shen S, Xiao H,Li G, Wang M and Mei J: Knockdown of SOX12 expression inhibits the proliferation and metastasis of lung cancer cells. Am J Transl Res 9: 4003-4014, 2017.

12. Gu W, Wang B, Wan F, Wu J, Lu X, Wang H, Zhu Y, Zhang H, Shi G, Dai B and Ye D: SOX2 and SOX12 are predictive of prognosis in patients with clear cell renal cell carcinoma. Oncol Lett 15: 4564-4570, 2018.

13. Wan H, Cai J, Chen F, Zhu J, Zhong J and Zhong H: SOX12: A novel potential target for acute myeloid leukaemia. Br J Haematol 176: 421-430, 2017.

14. Livak KJ and Schmittgen TD: Analysis of relative gene expression data using real-time quantitative PCR and the 2(-Delta Delta C(T)) Method. Methods 25: 402-408, 2001.

15. Song X, Yang $\mathrm{W}, \mathrm{Wu} \mathrm{C}, \mathrm{Han} \mathrm{YM}$ and Lu Y: USP9X promotes the proliferation, invasion and metastasis of liver cancer cells through regulating the JAK2/STAT3 signaling. Oncol Lett 20: 2897-2905, 2020 .

16. Chen W, Zheng R, Zhang S, Zeng H, Fan Y, Qiao Y and Zhou Q: Esophageal cancer incidence and mortality in China, 2010. Thoracic Cancer 5: 343-348, 2014.

17. Wang X, Lu Q, Fei X, Zhao Y, Shi B, Li C and Chen H: Expression and Prognostic Value of Id-4 in patients with esophageal squamous cell carcinoma. Onco Targets Ther 13: 1225-1234, 2020.

18. Chen W, Zheng R, Baade PD, Zhang S, Zeng H, Bray F, Jemal A, $\mathrm{Yu}$ XQ and He J: Cancer statistics in China, 2015. CA Cancer J Clin 66: 115-132, 2016.

19. Zou S, Wang C, Liu J, Wang Q, Zhang D, Zhu S, Xu S, Kang M and He S: Sox12 is a cancer stem-like cell marker in hepatocellular carcinoma. Mol Cells 40: 847-854, 2017. 
20. Jiang T, Guan LY, Ye YS, Liu HY and Li R: MiR-874 inhibits metastasis and epithelial-mesenchymal transition in hepatocellular carcinoma by targeting SOX12. Am J Cancer Res 7: 1310-1321, 2017.

21. Sinner D, Kordich JJ, Spence JR, Opoka R, Rankin S, Lin SC, Jonatan D, Zorn AM and Wells JM: Sox 17 and Sox4 differentially regulate beta-catenin/T-cell factor activity and proliferation of colon carcinoma cells. Mol Cell Biol 27: 7802-7815, 2007.

22. Wu R, Liu Y, Zhao Y, Bi Z, Yao Y, Liu Q, Wang F, Wang Y and Wang $\mathrm{X}$ : m6A methylation controls pluripotency of porcine induced pluripotent stem cells by targeting SOCS3/JAK2/STAT3 pathway in a YTHDF1/YTHDF2-orchestrated manner. Cell Death Dis 10: 171, 2019.

23. Zhao Q, Liu Y, Wang T, Yang Y, Ni H, Liu H, Guo Q, Xi T and Zheng L: MiR-375 inhibits the stemness of breast cancer cells by blocking the JAK2/STAT3 signaling. Eur J Pharmacol 884: $173359,2020$.

24. Verstovsek S, Manshouri T, Quintás-Cardama A, Harris D, Cortes J, Giles FJ, Kantarjian H, Priebe W and Estrov Z: WP1066, a novel JAK2 inhibitor, suppresses proliferation and induces apoptosis in erythroid human cells carrying the JAK2 V617F mutation. Clin Cancer Res 14: 788-96, 2008.

25. Bollrath J and Greten FR: IKK/NF-kappaB and STAT3 pathways: Central signalling hubs in inflammation-mediated tumour promotion and metastasis. EMBO Rep 10: 1314-1319, 2009.
26. Yang Y, Zhou H, Liu W, Wu J, Yue X, Wang J, Quan L, Liu H, Guo L, Wang Z, et al: Ganoderic acid A exerts antitumor activity against MDA-MB-231 human breast cancer cells by inhibiting the Janus kinase 2/signal transducer and activator of transcription 3 signaling pathway. Oncol Lett 16: 6515-6521, 2018.

27. Zhou X, Yan T, Huang C, Xu Z, Wang L, Jiang E, Wang H, Chen Y, Liu K, Shao Z and Shang Z: Melanoma cell-secreted exosomal miR-155-5p induce proangiogenic switch of cancer-associated fibroblasts via SOCS1/JAK2/STAT3 signaling pathway. J Exp Clin Cancer Res 37: 242, 2018.

28. Chang R, Song L, Xu Y, Wu Y, Dai C, Wang X, Sun X, Hou Y, Li W, Zhan X and Zhan L: Loss of Wwox drives metastasis in triple-negative breast cancer by JAK2/STAT3 axis. Nat Commun 9: 3486, 2018.

29. Zhang W, Qiao B and Fan J: Overexpression of miR-4443 promotes the resistance of non-small cell lung cancer cells to epirubicin by targeting INPP4A and regulating the activation of JAK2/STAT3 pathway. Pharmazie 73: 386-392, 2018.

This work is licensed under a Creative Commons Attribution-NonCommercial-NoDerivatives 4.0 International (CC BY-NC-ND 4.0) License. 